\title{
Spontaneous Resolution of Multiple Hyperpigmented Lesions with Platelet Transfusion
}

Brenda S Castillo ${ }^{1}$ and Behyar Zoghi ${ }^{2 *}$

${ }^{1} T e x a s$ Tech University Health Sciences Center, Paul L. Foster School of Medicine, EI Paso, Texas, USA

${ }^{2}$ Methodist Hospital, Texas Transplant Institute, San Antonio Texas, USA

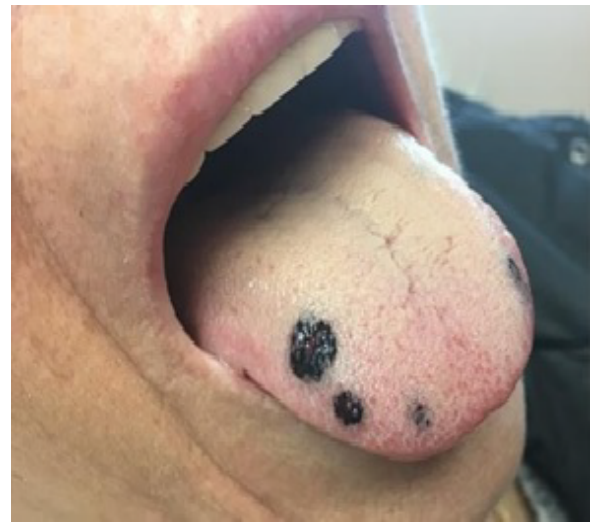

Figure 1: Shows lesions on the surface of her tongue.

Figure 2: After Platelet transfusion lesions removed completely.

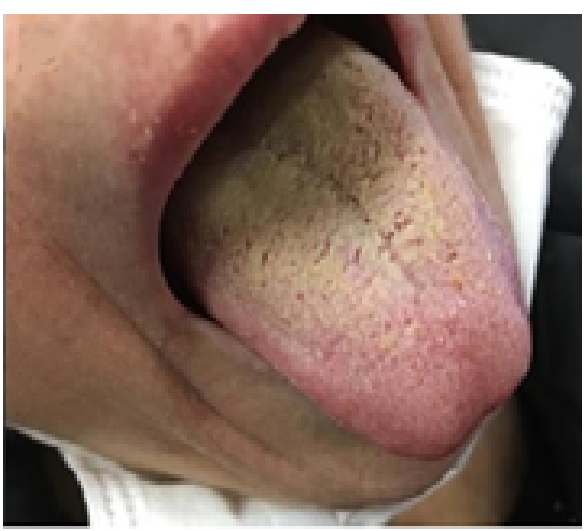

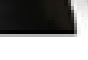

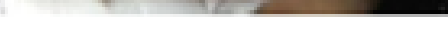

Keywords: Tongue; Black lesions; Cancer

\section{Clinical Image}

A 66-year-old female presents with a past medical history of hypertension, chronic back pain from injury following spinal fusion, and a family history positive for paternal grandfather with leukemia. Patient was diagnosed with Acute Myeloid Leukemia (AML) that originated from a background of myelodysplastic features. She initially presented with worsening fatigue and back pain, and was found to have pancytopenia around March 2017. Bone marrow biopsy showed AML 30\% blasts with complex cytogenetic abnormalities, including monosomy 7 and p53 mutations. Cerebrospinal Fluid (CSF) studies were negative. She was not considered as an allogeneic stem cell transplant candidate, and instead started on palliative chemotherapy. Patient tolerated well the combination of hypomethylating agents and Venetoclax. She had multiple admissions for neutropenic fever treated empirically with antibiotics. Upon clinic visit on January 2018, she presented with a low-grade fever, and was FLU positive. She was treated with Tamiflu, and completed the therapy by January 9, 2018. She presented again to the clinic on January 15, 2018 complaining of black, painless lesions on the surface of her tongue that were noted upon waking up (Figure 1). Complete Blood Count (CBC) showed White Blood Cell (WBC) 0.5, Hemoglobin (Hgb) 9.2, and Platelet count of 2. Dermatology was consulted, and it was planned to biopsy the lesions after the platelet transfusion were performed, given severe thrombocytopenia due to her chemotherapy regimen. Patient received the platelet transfusions and by the end of the day, upon completion of the transfusion, the lesions on her tongue completely resolved (Figure 2). It is possible, that the lesions were small hemorrhages. Most reported cases of oral hemorrhages have not presented in this manner, as they usually involve a wider area, and are confined to one spot rather than presenting at multiple sites, like in this case. This case is reported due to the rarity of the presentation of these lesions, and their complete, instantaneous resolution upon platelet transfusion. The aim of this article is to increase awareness among our fellow dermatologists and other physicians. 